\title{
The Analysis of the Current Situation and Development Prospect of Bolt Pretension
}

\author{
Zhao Pai-hang, Wang Ke-yin, Huang Hai-ying, Chen Yu-kun \\ Ordnance Engineering College, Shijiazhuang 050003, China
}

Keywords: Bolt; Pretightening force; Research status; Development prospects

\begin{abstract}
In order to guide the practice better with the study of pretightening force, this paper ansysed the preload theory research situation and application situation. Put forward the problems in the development of the existing pretightening force theory, it also analyzed the application status and development direction of torque wrench, and summarized the development needs and development direction of pretightening force theory. Preload data by using tensile test obtains each specification bolts, curve fitting using MATLAB software, the correlation function to find the bolt preload and specifications.
\end{abstract}

\section{Introduction}

Threaded connection is one of the most commonly, simple and effective connection , which is used in mechanical. The application field of the thread is extremely wide, in the field of mechanical transmission, thread also occupies a very important position. In the fluid material conveying and pressure transmission system, screw conveyor is also a common way of transmission. In order to enhance the rigidity, tightness and the locking capability of a screw connection and prevent slippage when the bolt subject to transverse load bolted, threaded connection during assembly to be preloaded. However, in order to prevent the bolt preload achieve the material yield limit point, but not too much preload. So you need to control the size of the preload within an appropriate range, both to meet the requirements and will not preload preload is too large to stress exceeds the limit.

Due to the thread's microstructure force deformation is difficult to observe,research on the forces of bolt is a computer simulation method thread stress analysis now, and draw conclusions as thread uneven force.

\section{Situation Bolt Preload Research}

2.1 The forces of threaded connection

Bolt connection is used to transmit the force and the machine parts connected to become an important part of the work as a whole. In general a variety of connecting bolts are low cost, but once bolted failure, the entire machine system might have disastrous consequences.

Thread failure is the main reason for failure of thread bolt, stress state and the influence of load distribution of screw thread strength to a large extent. The bolts in the situation including tensile, compression and shear stress in the connection machine. The shear stress on the thread connections stress distribution is not affected, do not do here analysis.

For the axial force of the bolt, the threads force is uneven. In the stretch-bolts work, the bolt is stretched, increasing the pitch of the threads; the nut is compressed, its thread pitch is reduced. Thus producing a poor pitch, leading to uneven force thread. Studies have shown that thread connection, approximately $1 / 3$ of the loadis strengthed on the first lap, descending, after the eighth lap of the thread almost unstressed.

\subsection{Preload theory}

"Mechanical Design Handbook" 5th Edition which the editor is CHENG Daxian pointed:Tightening torque $\mathrm{T}$ is used to overcome the drag torque screw of the thread deputy $\mathrm{T} 1$ and nuts with the connection (or washer) Friction torque between the bearing surface T2. Tightening torque is calculated as: 


$$
\begin{aligned}
& T=T_{1}+T_{2}=F \tan \left(\varphi+\rho_{v}\right) \frac{d_{2}}{2}+\frac{F \mu}{3} \times \frac{D_{w}^{3}-d_{0}^{3}}{D_{w}^{2}-d_{0}^{2}}=K F d \\
& K=\frac{d_{2}}{2 d} \tan \left(\varphi+\rho_{v}\right)+\frac{\mu}{3 d} \times \frac{D_{w}^{3}-d_{0}^{3}}{D_{w}^{2}-d_{0}^{2}}
\end{aligned}
$$

In the formula:d is nominal diameter of thread,F is preload,d2 is pitch diameter, ${ }^{\varphi}$ is thread lead angle, $\rho_{v}$ is thread equivalent friction angle, ${ }^{\mu}{ }_{v}$ is thread equivalent, $\mu_{\text {is the friction factor between }}$ the nut and the bearing surface, $\mathrm{K}$ is tightening torque coefficient.

Tightening torque coefficient responses the relationship between bolting tightening torque applied during and preload. It is the tighten process the most not easy to determined factor. Recommended values of $\mathrm{K}$ are shown in Table 1.

Table 1 Tightening torque coefficient $\mathrm{K}$

\begin{tabular}{ccc}
\hline \multirow{2}{*}{ Friction surface state } & \multicolumn{2}{c}{$\mathrm{K}$} \\
\cline { 2 - 3 } & Lubricating & No lubricating \\
\hline Surface finishing & 0.10 & 0.12 \\
General machined surface & $0.13 \sim 0.15$ & $0.18 \sim 0.21$ \\
Surface oxidation & 0.20 & 0.24 \\
Galvanized surface & 0.18 & 0.22 \\
Drying rough surface & - & $0.26 \sim 0.30$ \\
\hline
\end{tabular}

The calculation of preload $F$ is following:

Carbon steel bolts:

$$
F=(0.6 \sim 0.7) \sigma A
$$

Alloy steel bolts:

$$
F=(0.5 \sim 0.6) \sigma A
$$

In the formula: $\sigma$ is bolt material yield limit, $A$ is bolt stress area.

$$
\begin{aligned}
& A=\frac{\pi}{4}\left(\frac{d_{2}+d_{3}}{2}\right)^{2} \\
& d_{3}=d_{1}-\frac{H}{6}
\end{aligned}
$$

In the formula: $d_{1}$ is external thread trails, $d_{2}$ is external thread diameter, $d_{3}$ is calculate the diameter of thread, $H$ is original triangular thread height.

Sure there are other according to use and work method of determining pre tightening force, pressure vessel with flange bolt connection at the same time by the pretightening force and axial working load as an example, consider the following four points to determine the bolt tightening torque when: ensure that the bolt strength, which does not allow the loss of the connecting body because of excessive tightening torque result the bolt yield failure and function; II ensure thread strength, that is not because of excessive tightening torque resulting thread drop phenomenon; third, to ensure that the intensity of the flange plate, that is not because of excessive tightening torque causes flange will collapse; the ensuring performance of gasket, washer, not only to guarantee the tightening torque is too small will lead to leakage, also not because of the tightening torque due to the large gasket crushed, resulting in leakage of pressure after; out four kinds of situation tightening torque values are compared to determine the minimum value is the method of the tightening torque.

2.3 Preload control method.

Preload is too large or too small, there will be harm. So its size and precision are very vital. So the control of preload becomed one of the important issues on threaded connection. Commonly used methods:(1) Torque method;(2) Rotation Method;(3) Bolt hydraulic tension control method;(4) Special washers law etc.

Among them, The first two methods are more common. Torque method is based on the formula (1) by controlling the size of the torque $\mathrm{T}$ and then get the size of the preload force $\mathrm{F}$. The tightening torque coefficient $\mathrm{K}$ in Equation (1) according to the friction between the surface state of thread 
deputy be set. K-value error will lead directly to preload the error. When using a torque preload method, you need to use the proper tools such as a torque wrench to control the tightening torque of size. Therefore, the torque wrench accuracy error will also lead to increased preload error.

Angle control method is to achieve indirect control of preload according to the ratio between the rotation angle and the elongation of the bolt when tightening the nut. Assuming the object is a rigid connecting member and the bolt in the elastic deformation region, if the thread pitch for t, The relationship between the nut and the elongation of the angle of rotation is:

$$
\Delta l=\frac{\phi t}{360^{\circ}}
$$

When using this method, you need to compress the torque applied to eliminate the errors caused by the connection of the gap, and then start measuring the angle of rotation.

Relations with the bolt preload $\mathrm{F}$ and elongation is:

$$
F=\frac{\Delta l}{l} E A
$$

In the formula:l is bolt length, $\mathrm{E}$ is elastic modulus of the material,A is the average cross-sectional area of the bolt.

The formula (7) into equation (8) it follows that the relationship between the preload and the angle the formula:

$$
F=\frac{\phi t}{l \times 360^{\circ}} E A
$$

Therefore, within the elastic deformation range bolt, nut preload function of angle, by accurately controlling the rotation angle of the nut preload can be controlled. Error of this method is mainly due to the error caused by the elongation of the bolt. The other two methods are rarely used, are not discussed here.

2.4Situation preload application.

When replacing the family car tire, tighten the nuts are usually hand-tighten the foot does not move after then look tight stops. This method is commonly used in many car owners only, and even many professional repairman automotive factory this method is also used. The size of such methods preload applied entirely on the experience to be, for different operators, the tightening torque applied is different, this will lead to some not on tight, and some have more than the preload limit or even damage the thread, which gives cars has security implications, reducing the life of the tire nut.

Whether it is in the works or in life, using the torque angle method or Control Act few bolts, mostly relying on experience to grasp the size of torque, this will lead to less accurate preload applied to equipment or nuts security risk, reduce the life of equipment parts, reducing its efficiency, could lead to serious accidents.

\section{Problems and Development Needs}

\subsection{Problems}

3.1.1Bolt preload influenced by many factors.

In theory bolt preload process, there is some resistance to the primary, bolting is widely used, there are all kinds of bolts, a wide range of operating conditions, a variety of equipment, etc., need to be considered factors very much, forming a common theoretical formula has some difficulties. Further, failure of the bolt is generally from plastic deformation of thread begins, the deformation occurs in the bolts and nuts tight junction, the device can not be observed with a slight change material, many studies are based on the results of various simulation software based on the theory Research work has some difficulties from the test to proceed.

Preload threaded fasteners in a big gap between the industry, it is difficult to form a unified standard. For example, in the shipbuilding industry, some strong technical force equipment company to select the connection bolt tightening torque all with their own set of standards. As specializing in the development and production of highly flexible couplings and drive shafts VULKAN Germany and CENTA products company, will provide users with the appropriate 
tightening torque values bolting products for users.

3.1.2Theory in practice is not strong guidance.

Within a certain error range, based on the existing bolt preload theory can calculate the size of preload, using a torque wrench torque can be applied accurately. However, in the field of engineering, related theory for practical guidance is not strong, focus on efficiency under the premise, mostly controlled by the operator experience to preload size.

Currently, a number of technical issues have yet to fully grasp thread. Problems such as tightness, relax off the problem, test the strength of the design issues and problems. Practicality preload theory is not strong enough, the size of the required preload different conditions for different specifications of the bolt is no corresponding standard. Even in the case of a high precision torque wrench can not be accurately applied torque on the bolt, resulting in decrease in actual effect screwing. In many parts often occur after long-running loose bolts or fatigue damage phenomenon.

3.1.3The application is not wide enough torque wrench.

Torque wrench is a way to read and control the tightening torque wrench. There are a wide range of torque wrenches on the market, the type of relatively complete. There are pneumatic, hydraulic, electrical, mechanical. Pneumatic and hydraulic pumps and hydraulic pumps come with pressure and other ancillary equipment for fixed factory floor work, the whole equipment is relatively bulky, inconvenient to move, not suitable for field work. Electrical and mechanical advantages have small and flexible, easy to operate, suitable for field mobile operations. However, the need for electric batteries as a power. A direct result of their decline in accuracy when the battery power is low. Mechanical precision is lower failure rate, the impact of maintenance efficiency. In addition, high precision torque wrench generally higher prices. Low price and can not guarantee the accuracy of the torque wrench. This has hindered the application of a torque wrench.

At present, the torque wrench on the market has the precision and price to overwhelming difficulties, wrench high precision is usually digital display torque wrench, the majority of imported products, prices generally higher. Lower prices for torque wrench structure pointer and simple, the disadvantage is the low precision, difficult to satisfy the requirement of use. This is one reason for torque wrench in related fields of application is not wide enough.

3.2 Development needs

3.2.1Safe and reliable reference manual preload.

Existing preload selected references, such as corporate standards, formulas, etc. Most of the existence of the safety factor is too high leading torque chosen too small problem. In the field of mechanical engineering, the need both to ensure the lock, seal or fastening requirements threaded connection and can meet the performance requirements of the material. Avoid the blind pursuit of security, but the loss of mechanical properties, of course, but also to ensure a certain degree of safety factor ${ }^{[8]}$. Research and development of new products should be developed at the same time the details of the product. Contains preload reference value products threaded fasteners. Help to reduce the failure rate of products, improve the reliability of the equipment.

3.2.2Universal preload theory.

For ordinary standard bolts and nuts. Combined with the application of a torque wrench, you need to preload universal theory for support. Develop a more practical reference manual bolt preload. Can also be related to preload data in tabular form to organize, in the form of national standards to guide the production. Only practical, it is more conducive to the promotion, to be accepted..

3.2.3Economic and practical torque wrench.

On the basis of the existing torque wrench, torque wrench to further simplify the structure, reducing additional equipment. Such as pneumatic and hydraulic pump and hydraulic pump must be equipped with a direct product positioning to a fixed workshop, limiting its not move easily, flexibility is greatly reduced. Simple structure and high precision torque wrench, wrench reduce manufacturing costs, and simplify the process of operating wrenches, torque wrenches allow more civilians, mass. 


\section{Summary}

Threaded connection is the most widely used engineering mechanical connection. Proper preload will help threaded connection reliability and compactness, research and development of general theory preload will bring efficiency engineering production, bring convenience to people's lives, development and progress for the machinery industry to provide protection.

\section{References}

[1] Chai Jixin,Wang Enfeng,Sun Yingect. The Fixed Value Type Torque Wrench Automatic Verification Device[J]. Industrial Measurement,2009,(4):32-33.

[2] Chen Xiaoyan,Hu Shengqing,Wu Junting. Application of SPI in Digital Electronic Torque Wrench[J]. Electronics Quality,2004,(1) :11-13.

[3] Cheng Daxian. Mechanical Design Handbook[M], 5th Edition. Chemical Industry Press,2007.

LEE J,BOOK. Development of a New Transformer Type Torque Sensor[J].Key Engineering Materiacs,2004,270 /271/272/273:662-667

[4] Leng Jun. Loading method bolt preload research[J], Electrical Equipment,2013, (2), 32-35.

[5] Lu Peng,Yu Le,Liu Ye ect. Elastic method to study the bolt pretightening force with the patch of light[J]. Quarterly Journal of mechanics,2013,(2),275-278.

[6] Liu Cheng. Application of Pointer Type Torque Wrench in Construction[J]. Harbour Engineer, 1997(3):46-48.

[7] Fuat Kucuk,Hiroki Goto, Hai-Jiao Guo.Artificial neural networks and inductance vector based sensorless torque esti mation in switched reluctance motor drive[C].Electrical Machines and Systems, ICEM S,Seoul South Korea,2007.

[8] STOLAR SKITA. Analysis of the Resistance to Motion in a Sliding Contact[ J]. Wear, 1994, 171( 1- 2) :203- 209.

[9] Uthman Baroudi,Samir Mekid,Abdelhafid Bouhraoua.Bolt tension monitoring system.US, 20130139604[P],2013.06.06.

[10] Wang Yuhua,Wang Xiao,Yang,Hongjuan ect.Servral Expanding Applications of Capacitive Technology[J]. Tool Engineering , 2011,(6): 110 -112.

[11] Wu Huaiming, Shang Kailun Xue Ling ect.Design of Digital Torque Wrench's Wireless Communication based on the Capacitive Technology[J]. Journal of Astronautic Metrology and Measurement, 2014, (1): 71-74.

[12] YEN D C,CHOU D C. Wireless communication: the next wave of Internet technology[J]. Technology in Society,2001,23( 2) :217-226.

[13] Yi Xiao,Lin Jianhui. Application of Intelligent System in the Bolt Fastening Torque Wrench[J]. Manufacturing Automation,2013,(23):150-152

[14] Zhang Qiongmin. Study on the Process of Engine Cylinder Head Bolt Tightening[J]. Auto Mobile Science \& Technology, 2003,(2):19-22.

[15] Zhu Ruoyan,Li Houmin. Pre Tighten Force and Fatigue Life of High Strength Bolt[J]. Journal of Hubei University of Technology,2004,(3): 135-136+141.

[16] Zhao Man. Control method of bolt pretightening force[J]. Water Conservancy and Electric Power Machinery,2006,(7), 36-38.

[17] Zeng Guohua. Design of Controllable Torque of the Electric Wrench[J]. Tool Engineering,2002, 36( 5) : 15- 17 